\title{
COEFFICIENTS ESTIMATES FOR SOME FAMILIES OF BI-BAZELEVIC OF TYPE ALPHA $(\alpha)$ ASSOCIATED WITH ERROR FUNCTIONS
}

\author{
AWOLERE IBRAHIM TUNJI ${ }^{1, *}$ AND ASIRU TAJUDEEN MOTUNRAYO
}

\footnotetext{
${ }^{1}$ Department of Mathematical Sciences, Ondo State University of Science and Technology, P.M.B, Okitipupa, Ondo State, Nigeria

${ }^{2}$ Department of Mathematics, Emmanuel Alayande College of Education, P.M.B 1010, Oyo, Oyo State, Nigeria

*Corresponding Author: awolereibrahim01@gmail.com
}

Received Apr. 26, 2019

\begin{abstract}
AвSTRACT. In the present investigation, the authors are focusing on new subclasses of bi-univalent functions of Bazilevic functions of type $\alpha$ defined in the open disk, which are associated with error functions. Furthermore, estimates on Taylor-Maclaurin coefficients $\left|a_{2}\right|$ and $\left|a_{3}\right|$ for the function in the new subclasses introduced were obtained. Fekete-Szego functional $\left|a_{3}-\mu a_{2}^{2}\right|$ belong to new subclasses were also established. varying various parameter involved several known or new results were derived.
\end{abstract}

2010 Mathematics Subject Classification. Primary 30A10; Secondary 26 D07.

Key words and phrases. analytic functions; coefficient estimate; Bazilevic functions; error functions.

\section{InTRODUCTION}

Let $A$ be the class of function of the form

$$
f(z)=z+\sum_{k=2}^{\infty} a_{k} z^{k}
$$

which are analytic in the open unit disk $U=\{z:|z|<1\}$ and normalized $f(0)=f^{\prime}-1=0$.

Recall that $\operatorname{Re} \frac{z f^{\prime}(z)}{f(z)}>0$ refers to as a starlike function while $\operatorname{Re}\left(1+\frac{z f^{\prime \prime}(z)}{f^{\prime}(z)}\right)>0$ refers to as convex function. Let $T_{E r}^{\alpha}$ denote the class of the form

$$
\operatorname{SErf}(z)^{\alpha}=\left(z+\sum_{k=2}^{\infty} \frac{(-1)^{k-1}}{(2 k-1)(k-1) !} a_{k} z^{k}\right)^{\alpha}
$$

where $\operatorname{Erf}$ be a normalized analytic function defined by

$$
\operatorname{Erf}(z)=\frac{\sqrt{\pi z}}{2} \operatorname{erf}(\sqrt{z})=z+\sum_{k=2}^{\infty} \frac{(-1)^{k-1}}{(2 k-1)(k-1) !} z^{k}
$$

which are analytic and obtain from

$$
\operatorname{erf}(z)=\frac{2}{\sqrt{\pi}} \int_{0}^{z} \exp \left(-t^{2}\right) d t=\frac{2}{\sqrt{\pi}} \sum_{k=2}^{\infty} \frac{(-1)^{k-1} z^{2 k+1}}{(2 k+1) k !} .
$$

DOI: 10.28924/APJM/6-15 
Form (3) and (4) refer to error functions defined by [2] and [15] respectively. Recently, [15] investigated Hankel determinant for subclass of analytic functions associated with error functions bounded by conical regions and obtained interesting results. Also, many researchers have been able to use error function in different direction for detailed see [3], [6], [8]. Now using Binomial expansion on (2), we obtain

$$
\operatorname{SErf}(z)^{\alpha}=z^{\alpha}-\frac{\alpha}{3} a_{2} z^{\alpha+1}+\left[\frac{\alpha}{10} a_{3}+\frac{\alpha(\alpha-1)}{18}\right] z^{\alpha+2}+\left[\frac{-\alpha}{42} a_{4}-\frac{\alpha(\alpha-1)}{30} a_{2} a_{3}-\frac{\alpha(\alpha-1)(\alpha-2)}{162} a_{2}^{3}\right] z^{\alpha+3}
$$

which yields

$$
\frac{\operatorname{SEr} f(z)}{z^{\alpha}}=1-\frac{\alpha}{3} a_{2} z+\left[\frac{\alpha}{10} a_{3}+\frac{\alpha(\alpha-1)}{18}\right] z^{2}+\left[\frac{-\alpha}{42} a_{4}-\frac{\alpha(\alpha-1)}{30} a_{2} a_{3}-\frac{\alpha(\alpha-1)(\alpha-2)}{162} a_{2}^{3}\right] z^{3} .
$$

Researchers like $[1,10,11,12,13,17]$ and the likes have used (6) to defined several classes of Bazilevic functions and their results are well documented and too voluminous to discuss.

It is well-known that every function $f \in S$ has an inverse $f^{-1}$ defined by

$$
f\left(f^{-1}(w)\right)=w, \quad\left(|w|<r_{0}(f), \quad r_{0}(f) \geq \frac{1}{4}\right) .
$$

It is easily seen from above that where

$$
\begin{gathered}
f^{-1}(f(z))^{\alpha}=z^{\alpha}, \quad(z \in U) \\
f\left(f^{-1}(w)\right)^{\alpha}=w^{\alpha}, \quad\left(|w|<r_{0}(f), \quad r_{0}(f) \geq \frac{1}{4}\right),
\end{gathered}
$$

where

$$
\frac{\operatorname{SErf}(z)^{\alpha}}{z^{\alpha}}=1+\alpha_{1} a_{2} A_{2} z+\left[\alpha_{2} A_{2}^{2} a_{2}^{2}-\alpha_{1} a_{3} A_{3}\right] w^{2}+\left[2 \alpha_{1} a_{2} a_{3}-\alpha_{1} a_{4} A_{4}-\alpha_{3} A_{2}^{3} a_{2}^{3}\right] w^{3}+\ldots
$$

where $\alpha_{1}=\alpha, \quad \alpha_{2}=\frac{\alpha(\alpha+1)}{2}, \alpha_{3}=\frac{\alpha(\alpha+1)(\alpha+2)}{3 !}$

$$
\frac{S \operatorname{Erg}(w)^{\alpha}}{w^{\alpha}}=1-\alpha_{1} a_{2} A_{2} w+\left[\alpha_{2} a_{3} A_{3}+\alpha_{2} A_{2}^{2} a_{2}^{2}\right] z^{2}+\left[\alpha_{1} a_{4} A_{4}+2 \alpha_{1} a_{2} a_{3}+\alpha_{3} A_{2}^{3} a_{2}^{3}\right] z^{3}+\ldots
$$

where $\alpha_{1}=\alpha, \alpha_{2}=\frac{\alpha(\alpha-1)}{2}, \alpha_{3}=\frac{\alpha(\alpha-1)(\alpha-2)}{3 !}, A_{2}=-\frac{1}{3}, \quad A_{3}=\frac{1}{10}, A_{4}=-\frac{1}{42}$.

A function $f(z) \in A$ is said to be bi-univalent function in $U$ if both $f(z)$ and $g(w)$ are both univalent $U$. Here we denote the class of bi-univalent function in $U$ by $\sum$.

The object of this paper is to introduce new subclass of bi-univalence of Bazilevic functions associated with error function of type $\alpha$ and to determine the first few coefficient bounds and their relevants connection to Fekete-Szego estimates []. Our techniques shall depend on the earlier one use by Awolere et al.[5], Oladipo [12] , Strivastava et al.[18,19], Frazin and Aouf [9] and Aouf et al.[4]. 


\section{LemMA AND Definitions}

For the purpose of the present investigation, the following Lemmas and definitions were needed.

Lemma 2.1 (see [14]) If a function $p \in P$ is given by

$$
p(z)=1+p_{1} z+p_{2} z^{2}+\ldots \quad(z \in U)
$$

then $\left|p_{k}\right| \leq 2, \quad k \in N$, where $p$ is a family of all function $P$, analytic in $U$, for which $p(0)=1$ and $\operatorname{Rep}(z)>$ $0, z \in U$.

Lemma 2.2 (see $[7,16])$ Let the function $\phi(z)$ given by

$$
\phi(z)=\sum_{k=1}^{\infty} C_{k} z^{k} \quad(z \in U)
$$

be convex in $U$. Suppose also that the function $h(z)$ given by

$$
h(z)=\sum_{k=1}^{\infty} h_{k} z^{k} \quad(z \in U)
$$

is holomorphic $U$, if

$$
h(z) \prec \phi(z)
$$

then $\left|h_{n}\right| \leq\left|C_{1}\right|, \quad(n \in N)$.

Definition 2.1: A function $S E r f^{\alpha}$ given by (5) is said to be in the class $T_{\Sigma}(\alpha, \beta)$ if it satisfies the following

$$
\left|\frac{\operatorname{SErf}(z)^{\alpha}}{z^{\alpha}}\right|<\frac{\beta \pi}{2}
$$

and

$$
\left|\frac{\operatorname{SErg}(w)^{\alpha}}{w^{\alpha}}\right|<\frac{\beta \pi}{2}
$$

where $0<\beta \leq 1$ and $\alpha>0$.

Definition 2.2: A function $S E r f^{\alpha}$ given by (5) is said to be in the class $T_{\Sigma}(\alpha, \beta)$ if it satisfies the following

$$
\operatorname{Re}\left(\frac{\operatorname{SErf}(z)^{\alpha}}{z^{\alpha}}\right)>\beta
$$

and

$$
\operatorname{Re}\left(\frac{\operatorname{SErg}(w)^{\alpha}}{z^{\alpha}}\right)>\beta
$$

where $0<\beta \leq 1$ and $\alpha>0$.

Definition 2.3: A function $S E r f^{\alpha}$ given by (5) is said to be in the class $T_{\sum}(\alpha, \beta, \theta)$ if it satisfies the following

$$
e^{i \theta}\left(\frac{\operatorname{SEr} f(z)^{\alpha}}{z^{\alpha}}\right) \prec p(z) \cos \theta+i \sin \theta
$$

and

$$
e^{i \theta}\left(\frac{\operatorname{SErg}(w)^{\alpha}}{w^{\alpha}}\right) \prec q(w) \cos \theta+i \sin \theta
$$

where $\theta \in\left(-\frac{\pi}{2}, \frac{\pi}{2}\right)$ and $\alpha>0$.

Example 2.1: If we set

$$
h(z)=\frac{1+A z}{1+B z}=h_{A, B}(z), \quad(-1 \leq A<B \leq 1)
$$


then we have

$$
T_{E} r \sum(\alpha, \theta)=T_{E r} \sum\left(\alpha, \theta, \frac{1+A z}{1+B z}\right)=T_{E} r \sum\left(\alpha, \theta ; h_{A, B}\right)
$$

in which $T_{E} r \sum\left(\alpha, \theta ; h_{A, B}\right)$ denotes the class of function $f \in \sum$ satisfying the following conditions

$$
e^{i \theta}\left(\frac{S E r f(z)^{\alpha}}{z^{\alpha}}\right) \prec\left(\frac{1+A z}{1+B z}\right) \cos \theta+i \sin \theta
$$

and

$$
e^{i \theta}\left(\frac{\operatorname{SErg}(w)^{\alpha}}{w^{\alpha}}\right) \prec\left(\frac{1+A w}{1+B w}\right) \cos \theta+i \sin \theta
$$

where $\theta \in\left(-\frac{\pi}{2}, \frac{\pi}{2}\right)$ and $\alpha>0$.

Example 2.2: If in Example 1, we set

$$
A=1-2 \gamma \quad(0 \leq \gamma<1) \text { and } B=-1
$$

that is if we put

$$
h(z)=h_{1-2 \gamma,-1}=\frac{1+(1-2 \gamma) z}{1-z}=h_{\gamma}(z) \quad(0 \leq \gamma<1)
$$

then we get

$$
T_{E} r \sum(\alpha, \theta ; h)=T_{E r} \sum\left(\alpha, \theta, \frac{1+(1-2 \gamma) z}{1-z}\right)=T_{E} r \sum\left(\alpha, \theta ; h_{\gamma}\right)
$$

in which $T_{E} r \sum\left(\alpha, \theta ; h_{\gamma}\right)$ denotes the class of function $f \in \sum$ satisfying the following conditions

$$
\operatorname{Ree}^{i \theta}\left(\frac{\operatorname{SErf}(z)^{\alpha}}{z^{\alpha}}\right)>\gamma \cos \theta
$$

and

$$
\operatorname{Re}^{i \theta}\left(\frac{\operatorname{SErg}(w)^{\alpha}}{w^{\alpha}}\right)>\gamma \cos \theta
$$

where $\theta \in\left(-\frac{\pi}{2}, \frac{\pi}{2}\right)$ and $\alpha>0$ isreal, and the function $g$ is given by (12).

\section{MAIN RESULTS}

Theorem 3.1: Let function $S E r f^{\alpha}$ be given by (5) be in the class $T_{\sum}(\alpha, \beta), \quad \alpha>0$, then

$$
\begin{gathered}
\left|a_{2}\right| \leq \frac{3 \beta \sqrt{2\left|B_{1}\right|}}{\alpha} \\
\left|a_{3}\right| \leq \frac{10 \beta\left|B_{1}\right|}{\alpha}+\left(\frac{\sqrt{5} \beta\left|B_{1}\right|}{\alpha}\right)^{2} .
\end{gathered}
$$

Proof: It follows from Definition 1 that

$$
\frac{S E r f(z)^{\alpha}}{z^{\alpha}}=[p(z)]^{2}
$$

where $p(z)$ and $q(w)$ in $P$ having the form

$$
p(z)=1+p_{1} z+p_{2} z^{2}+\ldots \quad q(w)=1+q_{1} w+q_{2} w^{2}+\ldots
$$


Now equating the coefficients of (25) and (26) we get

$$
\frac{\alpha}{3} a_{2}=\beta q_{1}
$$

and

$$
\frac{\alpha}{10} a_{3}+\frac{\alpha(\alpha-1)}{18} a_{2}^{2}=\beta p_{2}+\frac{\beta(\beta-1)}{2} p_{1}^{2}
$$

From (28) and (30) we have that

$$
\frac{\alpha(\alpha+1)}{18} a_{2}^{2}-\frac{\alpha}{10} a_{3}=\beta q_{2}+\frac{\beta(\beta-1)}{2} q_{1}^{2} .
$$

and that

$$
\frac{2 \alpha^{2}}{9} a_{2}^{2}=\beta^{2}\left[p_{1}^{2}+q_{1}^{2}\right] .
$$

Now, from (29), (31) and (33) it is evident that

$$
\frac{\alpha^{2}}{9} a_{2}^{2}=\beta\left(p_{2}+q_{2}\right)+\frac{(\beta-1) \alpha^{2}}{9 \beta} a_{2}^{2} .
$$

Thus

$$
\frac{\alpha^{2}}{9 \beta} a_{2}^{2}=\beta\left(p_{2}+q_{2}\right) .
$$

Since, by definition, $p(z), q(w) \subset h(U)$, by application of Lemma 2.2 in conjunction with the Taylor-Maclaurin expression (27) we find that

$$
\left|p_{n}\right|=\left|\frac{p^{n}(0)}{n !}\right|=\left|B_{1}\right|, \quad(n \in N)
$$

and

$$
\left|q_{n}\right|=\left|\frac{q^{n}(0)}{n !}\right|=\left|B_{1}\right|, \quad(n \in N) .
$$

Thus, by using (36) and (37) for coefficients $p_{2}$ and $q_{2}$, we immediately have

$$
\left|a_{2}\right| \leq \frac{3 \beta \sqrt{2\left|B_{1}\right|}}{\alpha} .
$$

This gives the bound on $\left|a_{2}\right|$ as asserted in (23).

Next, in order to find bound for $\left|a_{3}\right|$, we subtract (31) from (29) using (32) we obtain

$$
\frac{\alpha}{5} a_{3}-\frac{\alpha}{9} a_{2}^{2}=\beta\left(p_{2}-q_{2}\right)+\frac{\beta(\beta-1)}{2}\left[p_{1}^{2}-q_{1}^{2}\right] .
$$

It follows from (38) and (33) that

$$
\frac{\alpha}{5} a_{3}=\beta\left(p_{2}-q_{2}\right)+\frac{\alpha}{9} a_{2}^{2}
$$


Thus

$$
\frac{\alpha}{5} a_{3}=\beta\left(p_{2}-q_{2}\right)+\frac{\beta^{2}\left[p_{1}^{2}+q_{1}^{2}\right]}{2 \alpha}
$$

Then

$$
a_{3}=\frac{5 \beta\left(p_{2}-q_{2}\right)}{\alpha}+\frac{5 \beta^{2}\left[p_{1}^{2}+q_{1}^{2}\right]}{2 \alpha} .
$$

Applying Lemma 2.2, (36) and (37) for coefficients $p_{1}, p_{2}, q_{1}$ and $q_{2}$

$$
\left|a_{3}\right| \leq \frac{10 \beta\left|B_{1}\right|}{\alpha}+\left(\frac{\sqrt{5} \beta\left|B_{1}\right|}{\alpha}\right)^{2} .
$$

Theorem 3.2: Let function $S E r f^{\alpha}$ be given by (5) be in the class $T_{\Sigma}(\alpha, \beta), \quad \alpha>0$, then

$$
\begin{gathered}
\left|a_{2}\right| \leq \frac{3 \beta \sqrt{2\left|B_{1}\right|(1-\beta)}}{\alpha} \\
\left|a_{3}\right| \leq \frac{10(1-\beta)\left|B_{1}\right|}{\alpha}+\left(\frac{\sqrt{5}(1-\beta)\left|B_{1}\right|}{\alpha}\right)^{2}
\end{gathered}
$$

Proof: It follows from Definition 2 that

$$
\begin{aligned}
& \frac{\operatorname{SErf}(z)^{\alpha}}{z^{\alpha}}=\beta+(1-\beta) p(z) \\
& \frac{\operatorname{SErg}(w)^{\alpha}}{w^{\alpha}}=\beta+(1-\beta) q(w)
\end{aligned}
$$

where $p(z)$ and $q(w)$ in $P$ having the form

$$
p(z)=1+p_{1} z+p_{2} z^{2}+\ldots \quad q(w)=1+q_{1} w+q_{2} w^{2}+\ldots .
$$

Now equating the coefficients of (44) and (45) we obtain

$$
\begin{gathered}
-\frac{\alpha}{3} a_{2}=(1-\beta) p_{1}, \\
\frac{\alpha}{10} a_{3}+\frac{\alpha(\alpha-1)}{18} a_{2}^{2}=(1-\beta) p_{2}, \\
\frac{\alpha}{3} a_{2}=(1-\beta) q_{1},
\end{gathered}
$$

and

$$
\frac{\alpha(\alpha+1)}{18} a_{2}^{2}-\frac{\alpha}{10} a_{3}=(1-\beta) q_{2} .
$$

Equivalently

$$
-\frac{\alpha}{3(1-\beta)} a_{2}=p_{1}
$$




$$
\frac{\alpha}{10(1-\beta)} a_{3}+\frac{\alpha(\alpha-1)}{18(1-\beta)} a_{2}^{2}=p_{2},
$$

$$
\frac{\alpha}{3(1-\beta)} a_{2}=q_{1},
$$

and

$$
\frac{\alpha(\alpha+1)}{18(1-\beta)} a_{2}^{2}-\frac{\alpha}{10(1-\beta)} a_{3}=q_{2} .
$$

From (50) and (52) we have that

$$
p_{1}=-q_{1}
$$

and that

$$
\frac{2 \alpha^{2}}{9(1-\beta)^{2}} a_{2}^{2}=\left[p_{1}^{2}+q_{1}^{2}\right] .
$$

Also, from (51) and (53) it is evident that

$$
\left[\frac{\alpha(\alpha-1)}{18(1-\beta)}+\frac{\alpha(\alpha+1)}{18(1+\beta)}\right]=p_{2}+q_{2} .
$$

Thus

$$
\frac{\alpha^{2}}{9(1-\beta)} a_{2}^{2}=\left(p_{2}+q_{2}\right) .
$$

Upon simplification (57) yields

$$
a_{2}^{2}=\frac{9\left(p_{2}+q_{2}\right)(1-\beta)}{\alpha^{2}} .
$$

Applying Lemma 2.2, (36) and (37) once again for the coefficients $p_{2}$ and $q_{2}$ we obtain

$$
\left|a_{2}\right| \leq \frac{3 \beta \sqrt{2\left|B_{1}\right|(1-\beta)}}{\alpha}
$$

which gives the bound on $\left|a_{2}\right|$ as asserted in (23).

Next, in order to find bound for $\left|a_{3}\right|$, we subtract (53) from (51) using (51) we obtain

$$
\frac{\alpha}{5} a_{3}-\frac{\alpha}{9} a_{2}^{2}=(1-\beta)\left(p_{2}-q_{2}\right) .
$$

It follows from (59) that

$$
\frac{\alpha}{5(1-\beta)} a_{3}=\left(p_{2}-q_{2}\right)+\frac{\alpha}{9(1-\beta)} a_{2}^{2} .
$$

Thus

$$
\frac{\alpha}{5(1-\beta)} a_{3}=\beta\left(p_{2}-q_{2}\right)+\frac{\left[p_{1}^{2}+q_{1}^{2}\right](1-\beta)}{2 \alpha}
$$

which yields

$$
a_{3}=\frac{5\left(p_{2}-q_{2}\right)(1-\beta)}{\alpha}+\frac{5\left[p_{1}^{2}+q_{1}^{2}\right](1-\beta)^{2}}{2 \alpha^{2}} .
$$


Applying Lemma 2.2, (36) and (37) for coefficients $p_{1}, p_{2}, q_{1}$ and $q_{2}$

$$
\left|a_{3}\right| \leq \frac{10(1-\beta)\left|B_{1}\right|}{\alpha}+\left(\frac{\sqrt{5}(1-\beta)\left|B_{1}\right|}{\alpha}\right)^{2}
$$

Theorem 3.3: Let function $S E r f^{\alpha}$ be given by (5) be in the class $T_{\sum}(\alpha, \theta), \quad \alpha>0$, then

$$
\left|a_{2}\right| \leq \frac{3 \beta \sqrt{2\left|B_{1}\right| \cos \theta}}{\alpha}
$$

$$
\left|a_{3}\right| \leq \frac{10 \beta\left|B_{1}\right| \cos \theta}{\alpha}+\left(\frac{\sqrt{5} \beta\left|B_{1}\right| \cos \theta}{\alpha}\right)^{2}
$$

Proof:It follows from (17) and (18) that

$$
e^{i \theta}\left(\frac{\operatorname{SEr} f(z)^{\alpha}}{z^{\alpha}}\right)=p(z) \cos \theta+i \sin \theta
$$

and

$$
e^{i \theta}\left(\frac{\operatorname{SErg}(w)^{\alpha}}{w^{\alpha}}\right)=q(w) \cos \theta+i \sin \theta
$$

where $p(z)$ and $q(w)$ in $P$ having the form

$$
p(z)=1+p_{1} z+p_{2} z^{2}+\ldots \quad q(w)=1+q_{1} w+q_{2} w^{2}+\ldots .
$$

respectively. Now equating the coefficients of (65) and (66) we get

$$
e^{i \theta}\left[\frac{\alpha}{10} a_{3}+\frac{\alpha(\alpha-1)}{18} a_{2}^{2}\right]=p_{2} \cos \theta
$$

$$
\begin{gathered}
\frac{e^{i \theta} \alpha}{3} a_{2}=q_{1} \cos \theta \\
e^{i \theta}\left[\frac{\alpha(\alpha+1)}{18} a_{2}^{2}-\frac{\alpha}{10} a_{3}\right]=q_{2} \cos \theta .
\end{gathered}
$$

From (67) and (69) we observe that

$$
p_{1}=-q_{1}
$$

and that

$$
\frac{2 \alpha^{2}}{9(1-\beta)^{2}} a_{2}^{2}=\left[p_{1}^{2}+q_{1}^{2}\right] e^{-i \theta} \cos \theta
$$

Also, from (68) and (70) it is evident that Thus

$$
\frac{\alpha^{2}}{9} a_{2}^{2}=\left(p_{2}+q_{2}\right) e^{-i \theta} \cos \theta
$$


Upon simplification (73) yields

$$
a_{2}^{2}=\frac{9\left(p_{2}+q_{2}\right) e^{-i \theta} \cos \theta}{\alpha^{2}}
$$

Applying Lemma $2.2 p_{2}$ and $q_{2}$ we obtain

$$
\left|a_{2}\right| \leq \frac{3 \beta \sqrt{2\left|B_{1}\right| \cos \theta}}{\alpha}
$$

which gives the bound on $\left|a_{2}\right|$ as asserted in (63).

Next, in order to find bound for $\left|a_{3}\right|$, we subtract (70) from (68) using (72) we have

$$
\frac{\alpha e^{i \theta}}{5} a_{3}=\left(p_{2}-q_{2}\right) \cos \theta+\frac{\left[p_{1}^{2}+q_{1}^{2}\right] e^{-2 i \theta} \cos ^{2} \theta}{2 \alpha} .
$$

Equivalently

$$
a_{3}=\frac{5\left(p_{2}-q_{2}\right) e^{-i \theta} \cos \theta}{\alpha}+\frac{5\left[p_{1}^{2}+q_{1}^{2}\right] e^{-2 i \theta} \cos ^{2} \theta}{2 \alpha^{2}} .
$$

Applying Lemma 2.2 for coefficients $p_{1}, p_{2}, q_{1}$ and $q_{2}$

$$
\left|a_{3}\right| \leq \frac{10\left|B_{1}\right| \cos \theta}{\alpha}+\left(\frac{\sqrt{5}\left|B_{1}\right| \cos \theta}{\alpha}\right)^{2}
$$

which complete the proof of the theorem.

Corollary 3.1: Let function $f^{\alpha} \in T_{\Sigma}(\alpha, 0)$ then we have that

$$
\left|a_{2}\right| \leq \frac{3 \sqrt{2\left|B_{1}\right|}}{\alpha}
$$

and

$$
\left|a_{3}\right| \leq \frac{10\left|B_{1}\right|}{\alpha}+\left(\frac{\sqrt{5} \beta\left|B_{1}\right|}{\alpha}\right)^{2}
$$

Corollary 3.2: Let function $f^{\alpha} \in T_{\sum}(1,0)$ then we have that

$$
\left|a_{2}\right| \leq 3 \sqrt{2\left|B_{1}\right|}
$$

and

$$
\left|a_{3}\right| \leq 10\left|B_{1}\right|+5\left|B_{1}\right|^{2}
$$

Theorem 3.4: Let function $S E r f^{\alpha}$ be given by (5) be in the class $T_{\sum}\left(\alpha, \theta ; h_{A, B}\right), \quad \alpha>0$, then

$$
\begin{gathered}
\left|a_{2}\right| \leq \frac{3 \sqrt{2(A-B) \cos \theta}}{\alpha} \\
\left|a_{3}\right| \leq \frac{10(A-B) \cos \theta}{\alpha}+\left(\frac{\sqrt{5}(A-B) \cos \theta}{\alpha}\right)^{2}
\end{gathered}
$$

Corollary 3.1: Let function $f^{\alpha} \in T_{\sum}(\alpha, 0)$ then we have that

$$
\left|a_{2}\right| \leq \frac{3 \sqrt{2(A-B)}}{\alpha}
$$


and

$$
\left|a_{3}\right| \leq \frac{10(A-B)}{\alpha}+\left(\frac{\sqrt{5}(A-B)}{\alpha}\right)^{2}
$$

Corollary 3.2: Let function $f^{\alpha} \in T_{\sum}(1,0)$ then we have that

$$
\left|a_{2}\right| \leq 3 \sqrt{2(A-B)}
$$

and

$$
\left|a_{3}\right| \leq 10(A-B)+5(A-B)^{2}
$$

Theorem 3.5: Let function $S E r f^{\alpha}$ be given by (5) be in the class $T_{\sum}\left(\alpha, \beta ; h_{1-2 \varphi}\right), \quad \alpha>0$, then

$$
\begin{gathered}
\left|a_{2}\right| \leq \frac{3 \sqrt{4(1-\varphi) \cos \theta}}{\alpha} \\
\left|a_{3}\right| \leq \frac{20(1-\varphi) \cos \theta}{\alpha}+\left(\frac{2(1-\varphi) \cos \theta \sqrt{5}}{\alpha}\right)^{2}
\end{gathered}
$$

\section{Conclusions}

Varying various parameter involved the results presented in this paper would lead to various (new or known) results.

\section{REFERENCES}

[1] S. Abdul Halim, On a class of analytic functions involving the Salagean differential operator, Tamkang J. Math. 23(1992), 51-58.

[2] M. Abramowitz and I. A. Stegun, (Eds), Handbook of Mathematical functions with Formulas, raphs, and Mathematical Tables, Dover Publications Inc., New York, 1965.

[3] H. Alzer, Error function inequalities, Adv. Comput. Math. 33(2010), no.3, 349-379,

[4] M.K.Aouf, R.M.El-Ashwah and A.M. Abd-Eltawab, New subclasses of bi-unvalent functions involving Dziok-Strivastava operator, ISRN Math. Anal. 2013 (2013), Article ID 387178.

[5] Awolere I. T and Ibrahim-Tiamiyu S, New classes of bi-univalent pseudo starlike function using Alhindi-Darus generalized hypergeometric functions, J. Nigeria Ass. Math. Phys. 40 (2017), 65-72.

[6] D. Coman, The radius of starlikeness for error function, Stu. Uni. Babes-Bolyai Math., 36 (1991), no.2, 13-16.

[7] P. L. Duren, Univalent Functions, Grundlehren der mathematischen Wissenscha en, vol. 259, Springer-Verlag, New York, NY, USA, 1983.

[8] A. Elbert and A. Laforgia, The zeros of the complementary error function, Numer. Algorithms, 49(2008), no.1-4, 153-157.

[9] B. A Frasing and M. K. Aouf, New subclasses of bi-univalent functions, Appl. Math. Lett. 24 (2011), no. 9, 1569-1573.

[10] J. O. Hamzat, Coefficients bounds for Bazilevic funvtions associated with modified Sigmoid function, Asian Res. J. Math. (2017), 33818.

[11] A. T. Oladipo and D. Breaz, On a class of family of bazilevic functions, Acta Universitatis Apulensis, 24 (2010), 319-330.

[12] A. T. Oladipo, New subclasses of bi-univalent Bazilevic functions of type alpha involving Salagean derivative operator, Acta Universitatis Apulensis, 43 (2015), 33-43.

[13] T. O. Opoola, on a new subclass of univalent functions, Mathematica, 36 (1994), no. 2, 195-200.

[14] C. Pommerenke, Univalent functions, Vandenhoeck and Ruprecht, Gottingen. 1975.

[15] C. Ramachandran and K. Dhanalakshmi and L. Vanitha, Hankel determinant for a subclass of analytic functions associated with error functions bounded by conical regions, Int., J. Math. Anal. 11 (2017), 571-581.

[16] W. Rogosinski, On the coefficients of subordinate functions, Proc. London Math. Soc. (Ser. 2), 48 (1943), 48-82.

[17] M. Sidiq and K.O. Babalola, Coefficient bounds for certain Bazilevic maps, Mathematica, 59 (82) (2017), 124-133. 
[18] H.M. Strivastava, A.K. Mishira and P. Gochhayat, certain subclasses of analytic and bi-univalent functions, Appl. Math. Lett. 23 (2010), 1188-1192.

[19] H.M. Strivastava, G. Murugusundaramorthy and K. Vijaya, Coefficient estimates for some families of bi-univalent functions of the Ma-Minda type involving the Hohlov operators, J. Classical Anal. 2 (2) (2013), 167-181.

[20] P.D. Tuan, V.V. Anh, Radius of starlikeness and convexity for certain classes of analytic functions , J. Math. Anal. Appl. 6 (1978), 146-158. 